\title{
ASPECTOS EMOCIONAIS DE PORTADORES DE OSTEOARTROSE QUE FREQUENTAM UM PROGRAMA DE REABILITAÇÃO FISIOTERAPÊUTICA EM GRUPO
}

Denis Henrique Zorzan Barreto, Raíssa Puzzi Ladvig, Alessandra Madia Mantovani Fabri, Cristina Elena Prado Teles Fregonesi, Cintia Tiemi Gushiken, Mariana Romanholi Palma, Ellene Cristine Lopes de Oliveira, Renilton José Pizzol, Dalva Minonroze Albuquerque Ferreira.

Universidade Estadual Paulista - UNESP, Departamento de Fisioterapia, Presidente Prudente, SP. E-mail: denishzb@hotmail.com

\section{RESUMO}

Analisou-se o perfil emocional de um programa de reabilitação fisioterapêutica em grupo, em pacientes com Osteoartrose (OA). A amostra integrou 25 pacientes com idade maior ou igual a 18 anos, ambos os sexos, diagnosticados com OA. Foram coletados dados pessoais e antropométricos, e aplicados questionários avaliando dor, qualidade de vida, ansiedade, depressão, níveis de estresse e autoestima dos pacientes. A média da idade foi $61,24 \pm 11,32$. As médias no questionário HAD foram, para ansiedade $13,90 \pm 2,18$, e depressão $14,66 \pm 1,52$. O LIPP variou entre $40 \%$ a $60 \%$ na fase II e III de sintomas estressores, no questionário SF-36 no aspecto emocional a média foi $34,65 \pm 40,22$ e no Rosenberg a autoconfiança teve média 16,32 $\pm 2,98$ e autodepreciação com 14,56 $\pm 4,05$. Este estudo mostrou que os sintomas de ansiedade e depressão estão presentes no processo do envelhecimento juntamente com a OA. Diante disso, torna-se indispensável à avaliação e o tratamento adequado do perfil emocional desses pacientes.

Palavras-chave: Osteoartrite, sintomas afetivos, qualidade de vida, terapia por exercício.

\section{EMOTIONAL ASPECTS OF OSTEOARTHRITIS CARRIERS THAT FREQUENT A GROUP PHYSIOTHERAPY REHABILITATION PROGRAM}

\begin{abstract}
The emotional profile of a group physiotherapeutic rehabilitation program was analyzed in patients with Osteoarthrosis (OA). The sample consisted of 25 patients aged 18 years or older, both sexes, diagnosed with OA. Personal and anthropometric data were collected, and questionnaires were evaluated evaluating pain, quality of life, anxiety, depression, stress levels and self-esteem of the patients. The mean age was $61.24 \pm 11.32$. The means in the HAD questionnaire were, for anxiety $13.90 \pm 2.18$, and depression $14.66 \pm$ 1.52. The LIPP ranged from $40 \%$ to $60 \%$ in stage II and III of stressor symptoms, in the SF-36 questionnaire the emotional aspect was $34.65 \pm 40.22$ and in Rosenberg the self-confidence had a mean of $16.32 \pm 2.98$ and self-depreciation with $14.56 \pm 4.05$. This study showed that the symptoms of anxiety and depression are present in the aging process along with OA. Therefore, it becomes indispensable to the evaluation and appropriate treatment of the emotional profile of these patients.
\end{abstract}

Keywords: Osteoarthritis, affective symptoms, quality of life, exercise therapy.

\section{INTRODUÇÃO}

A osteoartrose $(O A)$ é uma doença crônico-degenerativa caracterizada pela perda progressiva da cartilagem articular, alteração do osso subcondral e inflamação da sinovia. Pode ser classificada como primária, quando a causa é idiopática, hereditária e secundária, quando é provocada por traumas ou fraturas ${ }^{1,2}$.
No Brasil, mais da metade da população apresenta essa afecção, sendo fator impactante na vida dos trabalhadores. Isso implica na incapacidade laboral e saúde mental, que pode levar a problemas psicológicos como depressão, ansiedade e transtornos ${ }^{3,4}$, uma vez que a $O A$ aumenta o risco do aparecimento de diversas comorbidades $^{5}$. 
Com o envelhecimento da população, as doenças crônico-degenerativas acompanham esse processo natural, exponencialmente, tornando-se um papel importante na saúde básica ${ }^{6}$. Com o avançar da idade, a prevalência dessas condições crônicas e incapacidade física vêm crescendo, assim, é necessário avaliar os impactos da saúde na qualidade de vida destes indivíduos ${ }^{1}$.

O envelhecimento não está primordialmente ligado a Doenças Crônicas Não Transmissíveis (DCNT), porém a prevenção da saúde, controle da pressão arterial, diabetes e do peso podem ser prevenidos para não desencadear tal condição clínica $^{7}$. Com isso a prática da atividade física é indicada como forma de prevenção para o não surgimento ou agravamento dessa condição. Apesar da atividade física não interromper o processo de envelhecimento e desgastes destas articulações, ela pode reduzir os sintomas e impactos do efeito fisiológico desse processo, pode também promover melhora da saúde, qualidade de vida e capacidade funcional e diminuição dos sinais e sintomas da $\mathrm{OA}^{8,9}$.

Uma alternativa para o tratamento das DCNT pode ser o uso de fármacos para controlar e reduzir os sintomas, e medidas não farmacológicas como exercícios físicos e fisioterapia são as mais indicadas comumente para a melhora da qualidade de vida ${ }^{10}$.

A fisioterapia, associada aos cuidados médicos, pode minimizar o impacto da OA na saúde e evitar complicações que levam à incapacidade funcional, por meio de tratamento que objetive a redução do quadro álgico, inflamatório, diminuição da força muscular e diminuição da amplitude de movimento ${ }^{11}$. Assim, considerando o impacto dos aspectos emocionais no agravamento dos sintomas da OA, o objetivo do presente estudo foi levantar o perfil emocional, baseado na avaliação dos sintomas da dor, qualidade de vida, ansiedade, depressão, níveis de estresse e autoestima, em pacientes com $O A$ que frequentam um programa de reabilitação fisioterapêutica em grupo.

\section{METODOLOGIA}

Trata-se de um ensaio clínico observacional não aleatorizado, desenvolvido em um centro de Fisioterapia e Reabilitação em Presidente Prudente, SP. Foi aprovado pelo Comitê de Ética em Pesquisa (CAAE:
69457717.1.0000.5402). Após concordarem, os pacientes assinaram o Termo de Consentimento Livre e Esclarecido.

A amostra foi composta por 25 pacientes com idade maior ou igual a 18 anos, de ambos os sexos, portadores de $\mathrm{OA}$, frequentadores de um programa de fisioterapia em grupo, duas vezes na semana, com duração de $55 \mathrm{~min}$, totalizando 16 sessões. Foram coletados nome e idade dos pacientes e questionado sobre a presença de comorbidades, medicamentos e substâncias em uso e quanto a realização de atividades físicas. Para a antropometria, foi obtido o peso e altura dos pacientes por meio de balança digital com estadiômetro (Welmy ${ }^{\circledR}, \mathrm{W} 110 \mathrm{H}$, Brasil). Para o cálculo do índice de massa corporal (IMC), foi dividido o peso (em $\mathrm{Kg}$ ) pelo quadrado da altura (em metros), de acordo com a Organização mundial da Saúde ${ }^{12}$. Após, foram aplicados questionários para avaliação da dor, qualidade de vida, sintomas de ansiedade e depressão, níveis de estresse e autoestima.

O nível da dor foi avaliado em repouso, utilizando a escala visual analógica (EVA), uma escala unidimensional composta com números que variam de zero a 10 , sendo que o escore zero indica ausência de dor e dez denota dor insuportável ${ }^{13}$.

Utilizou-se o questionário SF-36 para avaliação de qualidade de vida, formado por 36 itens subdivididos em oito domínios: capacidade funcional, aspectos físicos, dor, estado geral de saúde, vitalidade, aspectos sociais, aspectos emocionais e saúde mental. A pontuação varia de zero a 100 , no qual zero corresponde ao pior estado geral de saúde e 100 ao melhor ${ }^{14}$.

Para a avaliação dos sintomas de ansiedade e depressão foi utilizado a escala Hospital Anxiety and Depression (HAD), composta por 14 questões. A pontuação varia de zero a 21 pontos, significando: 0 a 7 , improvável ansiedade ou depressão; 8 a 11, possível; e 12 a 21 pontos o paciente encontra-se com provável presença desses sintomas ${ }^{15}$.

Para a avaliação do nível de estresse e presença de sintomas físicos e ou psicológicos foi utilizado o Inventário de Lipp, composto por três quadros, referentes às fases do nível de estresse. O primeiro quadro (fase de alerta) corresponde aos sintomas das últimas 24 horas. 0 segundo (fase de resistência), referentes à última semana. E O terceiro quadro (fase de exaustão), correspondentes ao último mês. Sete itens ou 
mais assinalados na fase um, indica a fase de alerta; quatro itens ou mais na fase dois indicam a fase de resistência e nove itens ou mais na fase três indica que o respondente encontra-se em fase de exaustão ${ }^{16}$.

Utilizou-se a escala de Rosenberg, constituída por 10 afirmações que avalia a autoestima global. Para cada afirmação existem quatro opções de resposta (concordo totalmente $=4$, concordo $=3$, discordo $=2$ e discordo totalmente $=1$ ). A somatória dos 10 itens dará a cotação da escala, cuja pontuação total oscila entre 10 e 40 . A pontuação alta reflete uma autoestima elevada ${ }^{17}$.

Os dados foram apresentados por estatística descritiva por meio de média e desvio padrão para dados contínuos e prevalência com seu respectivo intervalo de confiança de $95 \%$ para dados categóricos. Foi adotado o nível de significância $p \leq 0,05$. Foi utilizado o software SPSS versão 13.0.

\section{RESULTADOS}

A idade média da amostra foi de $61,24 \pm 11,32$ anos. As demais características amostrais estão representadas na Tabela 1.

Os dados da avaliação psicométrica realizada pelo questionário $H A D$, inventário LIPP, questionário SF-36 e a escala de Rosenberg seguem descritos na Tabela 2.

Tabela 1. Caracterização da amostra de indivíduos com Osteoartrose, segundo idade, composição corporal e nível de dor $(n=25)$.

\begin{tabular}{lll}
\hline Dados descritivos & $\mathrm{n}$ & $\%(\mathrm{IC} 95 \%)$ \\
\hline Idade & & \\
$\leq 45$ & 2 & $8(0-18,63)$ \\
$46-60$ & 11 & $44(24,54-63,46)$ \\
$>60$ & 12 & $48(28,42-67,58)$ \\
\hline Sexo & & \\
Masculino & 6 & $24(7,26-40,74)$ \\
Feminino & 19 & $76(59,26-92,74)$ \\
\hline Índice de Massa Corporal & & \\
Normal & 3 & $12(0-24,74)$ \\
Sobrepeso & 10 & $40(20,80-59,20)$ \\
Obesidade & 12 & $48(28,42-67,58)$ \\
\hline Escala Visual Analógica & & \\
0 & 3 & $12(0-24,74)$ \\
1-3 & 8 & $32(13,71-50,29)$ \\
$4-6$ & 5 & $20(4,32-35,68)$ \\
$7-10$ & 9 & $36(17,18-54,82)$ \\
\hline
\end{tabular}

\section{DISCUSSÃO}

O propósito do presente estudo foi analisar os aspectos emocionais de portadores de OA submetidos a um programa de reabilitação fisioterapêutica. Na análise descritiva sobre idade, sexo, IMC e EVA, observou-se que a faixa etária variou entre 45 e 60 anos correspondendo a mais da metade dos pacientes, a predominância foi do sexo feminino, $48 \%$ pacientes de acordo com o IMC estão classificados como obesos e segundo o EVA a dor foi mais frequente entre os níveis 7 e 10 sendo classificada como dor intensa.

Os resultados obtidos por meio do questionário HAD no domínio ansiedade, 13 pacientes apresentaram uma provável ansiedade o que corresponde $40 \%$ da amostra. No domínio depressão, 8 apresentaram uma possível depressão o que corresponde $32 \%$ da amostra. No inventário LIPP os resultados variaram entre a fase II e fase III o que indica que os pacientes apresentaram sintomas referente por uma semana e durante os últimos 3 meses. Na escala de Rosenberg em ambos os domínios autoconfiança e autodepreciação, o escore ficou abaixo da média. No questionário SF-36 o domínio aspecto emocional apresentou escore inferior a $50 \%$ o que representa um comprometimento na vida destes pacientes. 
Tabela 2. Resultados dos questionários HAD, LIPP, SF36 e Escala de Rosenberg, de indivíduos com Osteoartrose $(n=25)$.

\begin{tabular}{|c|c|c|c|}
\hline & Média $\pm D P$ & $\mathrm{n}$ & $\%(I C 95 \%)$ \\
\hline \multicolumn{4}{|l|}{ HAD ansiedade } \\
\hline Improvável & $4,87 \pm 1,12$ & 8 & $32(13,71-50,29)$ \\
\hline Possível & $9,28 \pm 1,38$ & 7 & $28(10,40-45,60)$ \\
\hline Provável & $13,90 \pm 2,18$ & 10 & $40(20,80-59,20)$ \\
\hline \multicolumn{4}{|l|}{ HAD depressão } \\
\hline Improvável & $4,28 \pm 2,12$ & 14 & $56(36,54-75,46)$ \\
\hline Possível & $9,00 \pm 0,92$ & 8 & $32(13,71-50,29)$ \\
\hline Provável & $14,66 \pm 1,52$ & 3 & $12(0-24.74)$ \\
\hline \multicolumn{4}{|l|}{ LIPP (positivo) } \\
\hline Fase I & - & 6 & $24(7,26-40,74)$ \\
\hline Fase II & - & 15 & $60(40,80-79,20)$ \\
\hline Fase III & - & 11 & $44(24,54-63,46)$ \\
\hline SF36 & & Mínimo & Máximo \\
\hline Capacidade Vital & $30,92 \pm 16,83$ & 5 & 85 \\
\hline Aspectos Físicos & $9,00 \pm 20,25$ & 0 & 75 \\
\hline Dor & $29,11 \pm 16,65$ & 2 & 74 \\
\hline Estado Geral de Saúde & $52,78 \pm 20,46$ & 15 & 100 \\
\hline Vitalidade & $52,80 \pm 21,26$ & 5 & 85 \\
\hline Aspectos Sociais & $54,90 \pm 19,47$ & 0 & 100 \\
\hline Aspectos Emocionais & $34,65 \pm 40,22$ & 0 & 100 \\
\hline Saúde Mental & $61,80 \pm 24,23$ & 12 & 100 \\
\hline \multicolumn{4}{|l|}{ Rosenberg } \\
\hline Autoconfiança & $16,32 \pm 2,98$ & 10 & 20 \\
\hline Autodepreciação & $14,56 \pm 4,05$ & 8 & 20 \\
\hline Total & $30,84 \pm 6,49$ & 18 & 40 \\
\hline
\end{tabular}

$\mathrm{Na}$ terceira idade a depressão pode se manifestar em decorrência de diversas doenças clínicas como a $\mathrm{OA}$, o que implica na diminuição das atividades físicas diárias e a perda da autonomia, conforme Pimenta et al. ${ }^{18}$, o que corrobora com o presente estudo. De acordo com Leite et al. $^{19}$, foi verificado que o impacto da depressão é relevante uma vez que os pacientes depressivos estão mais susceptíveis a apresentarem quadro álgico crônico. Esse quadro pode ser um fator para a não adesão ao tratamento, tendo em vista que a dor limita o movimento podendo levar essa articulação ao desuso e consequentemente amplificar a dor.

Esse transtorno depressivo associado a doenças físicas pode desenvolver o surgimento de sintomas de ansiedade. Considerando que a OA é uma doença incapacitante, presumi-se que ela pode estabelecer um fator estressante e favorecer o aparecimento do mesmo. Tais alterações como tensão motora e dificuldade de concentração podem levar esses pacientes a um estado de fuga, esquiva e aumento da irritabilidade, conforme o estudo de Valença et al. $^{20}$.

A dor e suas complicações no estado mental em pacientes com $\mathrm{OA}$ são elementos importantes e afetam a qualidade de vida dos mesmos. Os resultados deste estudo indicam que há um índice inferior no domínio aspecto emocional do instrumento SF-36. Uma explicação pode ser que a dor promova dificuldades nas AVD's gerando uma dependência física, diminuição da mobilidade e, a integração social causada pelas incapacidades podem aumentar a ansiedade, o desânimo, intensificar os efeitos da OA e levar a piora da percepção do paciente em relação a saúde mental ${ }^{21}$.

$\mathrm{Na}$ literatura o questionário mais utilizado para a avaliação da depressão foi o Inventário de Depressão Beck, e para a ansiedade foi o Inventário de Ansiedade Traço-estado (IDATE). Portanto, uma limitação desse estudo, foi a escassez de estudos que associavam os 
questionários HAD e Rosenberg com a saúde mental do paciente. Outro fator limitante foi o tamanho reduzido ou a desistência da amostra e a avaliação de alguns pacientes que já participavam ou praticavam atividades físicas regulamente.

As vantagens deste estudo foram as utilizações dos questionários HAD e Rosenberg para a análise do perfil emocional dos pacientes na qual os questionários apresentaram confiabilidade e contribuição para a análise comportamental do estado mental desses pacientes, colaborando assim, para estudos futuros.

\section{CONCLUSÃO}

O presente estudo mostrou que os sintomas de ansiedade e depressão estão presentes no processo do envelhecimento juntamente com as DCNT como a OA. Diante disso, torna-se indispensável a avaliação e o tratamento adequado do perfil emocional desses pacientes.

\section{CONFLITO DE INTERESSE}

Os autores declaram não haver qualquer potencial conflito de interesse que possa interferir na imparcialidade deste trabalho científico.

\section{REFERÊNCIAS}

1. Alexandre TS, Cordeiro RC, Ramos LR. Fatores associados à qualidade de vida em idosos com osteoartrite de joelho. Fisioterapia e Pesquisa. 2008; 15(4): 326-32.DOI: https://doi.org/10.1590/S1809$\underline{29502008000400002}$

2. Takahashi F, Ladeira JP, Lima AM. Osteoartrite. In: Ribeiro ACM, Takahashi F. (Eds.). Principais temas em reumatologia para residência médica. 2.ed. São Paulo: Medcel; 2006. p.59-70.3.

3. Creamer $P$, Lethbridge-cejku $M$, Hochberg MC. Factors associated with functional impairment insymptomatic knee osteoarthritis. Brit SocRheumatol. 2000;8(2):490-6.DOI: https://doi.org/10.1093/rheumatology/39.5.490

4. Dominick KL, Ahern FM, Gold CH, Heller DA. Health-related quality of life among older adults with arthritis.Health Qual Life
Outcomes2004;2:5.DOI:

https://doi.org/10.1186/1477-7525-2-5

5. Santos NGB, Figueiredo Neto EM, Arêas GPT, Arêas FZS, LeiteHR, Ferreira MAC, et al. Capacidade funcional e qualidade devida em idosos com osteoartrose no município de Coari (AM).RevPesq Fis. 2012;2:107-20.

6.Kilsztajn S, Rossbach A, Câmara MB, Carmo MSN. Serviços de saúde, gastos e envelhecimento da população brasileira. RevBras Estudos População. 2003; 20(1).

7. Veras R. Envelhecimento populacional contemporâneo: demandas, desafios e inovações. Rev SaúdePública. 2009;43(3):54854.https://doi.org/10.1590/S0034$\underline{89102009000300020}$

8. Al-Nsour M, Zindah M, Belbeisi A, Hadaddin R, Brown DW, Walke H. Prevalence of selected chronic, noncommunicable disease risk factors in Jordan: results of the 2007 Jordan Behavioral Risk Factor Surveillance Survey. Prevchronicdis2012;9(25):1-9.

9. Meurer ST, Beneditti TRB, Mazo GZ. Fatores motivacionais de idosos praticantes de exercícios físicos: um estudo baseado na teoria da autodeterminação. EstudPsicol 2012;17(2):299303.DOI: $\quad$ https://doi.org/10.1590/S1413$\underline{294 \times 2012000200014}$

10. Hochberg $M C$, Altman RD, Brandt KD, Clark BM, Dieppe PA, Griffin MR, et al. Guidelines for the medical management of osteoarthritis. Arthritis Rheumatism. 1995;38(11):1541-46.DOI: https://doi.org/10.1002/art.1780381104

11. Marques AP, Kondo A. A fisioterapia na osteoartrose: uma revisão da literatura. RevBrasReumatol. 1998; 38(2):83-90.

12. Rezende FAC, Rosado LEFPL, Franceschinni SCC, Rosado GP, Ribeiro RCL. Aplicabilidade do índice de massa corporal na avaliação da gordura corporal. Rev Bras Med Esp. 2010;16(2):90-4. DOI: $\quad$ https://doi.org/10.1590/S1517$\underline{86922010000200002}$

13. Price DD, McGrath PA, Rafii A, Buckingham $B$. The validation of visual analogue scales as ratio scale measures for chronic and experimental 
pain.

Pain. 1983;17(1):45-56.DOI: https://doi.org/10.1016/0304-3959(83)90126-4

14.Ciconelli, R. M. et. al. Tradução para a língua portuguesa e validação do questionário genérico de avaliação de qualidade de vida SF36 (Brasil SF36).RevBrasReumatol.1999;39(3):143-50.

15. Botega, N. J. et al. Transtornos do humor em enfermaria de clínica médica e validação de escala de medida (HAD) de ansiedade e depressão, Revista de Saúde Pública. 1995;29(5):355-63.DOI:

https://doi.org/10.1590/S0034-

89101995000500004

16.Lipp, M. E. N.; Guevara, A. J. H. Validação empírica do inventário de sintomas de stress. Estudos Psicol.1994; 11(3):43-9.

17.Hutz, C. S.; Zanon, C. Revisão da adaptação, validação e normatização da escala de autoestima de Rosenberg. Avaliação Psicol.2011;10(1):41-9.

18.Pimenta FAP, Simil FF, Tôrres HOG, Amaral CFS, Rezende CF,Coelho TO, et al. Avaliação da qualidade de vida deaposentados com a utilização do questionário Sf-36. RevAssocMed Bras. 2008;54:55-60.DOI:

https://doi.org/10.1590/S0104-

$\underline{42302008000100021}$

19. Leite AA, Costa AJG, Lima BAM, Padilha AVL, AlbuqueruqeEC,Marques CDL. Comorbidades em pacientes com osteoartrite:frequência e impacto na dor e na função física. RevBrasReumatol. 2011;51:118-23.DOI:

https://doi.org/10.1590/S0482-

50042011000200002

20. Valencia AM, Freire R, Santos LM, Sena IM, Campinho JL,Martuscello Neto $C$, et al. Transtornos de ansiedade e depressão em pacientes de ambulatório de clínica médica.RBM. 2008;65:12-7.

21. Alyne HF, Priscilla BGG, Nara RRASD, Ricardo EASD, Ricado CP, Regina CBS. Investigação da ansiedade, depressão e qualidade de vida em pacientes portadores de osteoartrite no joelho: um estudo comparativo. RevBrasReumatol. 2015;55(5):434-38.DOI: https://doi.org/10.1016/j.rbr.2015.03.001
Recebido para publicação em 10/08/2018

Revisado em 06/09/2018

Aceito em 09/09/2018 